\title{
Nutritional depletion in relation to respiratory and peripheral skeletal muscle function in out-patients with COPD
}

\author{
M.P.K.J. Engelen, A.M.W.J. Schols, W.C. Baken, \\ G.J. Wesseling, E.F.M. Wouters
}

Nutritional depletion in relation to respiratory and peripheral skeletal muscle function in out-patients with COPD. M.P.K.J. Engelen, A.M.W.J. Schols, W.C. Baken, G.J. Wesseling, E.F.M. Wouters. CERS Journals Ltd 1994.

ABSTRACT: Although increasing attention has been paid to nutritional aspects in chronic obstructive pulmonary disease (COPD), limited information is available regarding the prevalence and consequences of nutritional depletion in a random out-patient COPD population.

We studied body composition in relation to respiratory and peripheral skeletal muscle function in 72 COPD patients (mean (SD) forced expiratory volume in one second $\left(\mathrm{FEV}_{1}\right) 53$ (15) \% predicted), who came to the lung function laboratory for routine lung function measurements. Patients were characterized by the degree of body weight loss and fat-free mass depletion. According to this definition, $14 \%$ of the group suffered from both loss of body weight and depletion of fat-free mass, whereas $7 \%$ had one of these conditions.

We found that tissue depletion was concomitant with lower values for respiratory and peripheral skeletal muscle strength $(46.0(27.2)$ vs $77.1(29.8) \mathrm{kg})$, and a significantly lower transfer coefficient for carbon monoxide (Kco 64.9 (16.2) vs 81.9 (24.5) \% pred). Stratification by KCo $(<60 \%$ vs $>\mathbf{8 0} \%)$ also revealed significantly lower values for fat-free mass and higher values for intrathoracic gas volumes, total lung capacity (TLC) and residual volume (RV) in the group with a $\mathrm{KCO}<60 \%$ pred. Analysis of covariance, taking fat-free mass as covariate, indicated an independent contribution of Kco on maximal inspiratory mouth pressure (PImax) but not on peripheral skeletal muscle strength.

It is concluded that a substantial number of COPD out-patients suffer from nutritional depletion, preferentially affecting peripheral skeletal muscle function. Eur Respir J., 1994, 7, 1793-1797.
Dept of Pulmonology, University of Limburg, Maastricht, The Netherlands

\author{
Correspondence: A.M.W.J. Schols \\ Dept of Pulmonology \\ University of Limburg \\ P.O. Box 5800 \\ 6202 AZ Maastricht \\ The Netherlands
}

\begin{abstract}
Keywords: Airflow obstruction chronic obstructive pulmonary disease muscles
\end{abstract}

nutrition

Received: July 301993

Accepted after revision July 171994
Recently, increasing attention has been paid to nutritional aspects in chronic obstructive pulmonary disease (COPD), since weight loss and a low body weight correlate with increased morbidity and a poor prognosis $[1,2]$.

Since 1981 several studies have investigated the prevalence of nutritional depletion in COPD. Depending on the patient population analysed, 20-70\% of the COPD patients were found to be underweight [3-8]. All the reported studies were performed in selected populations, such as hospitalized patients, patients entering rehabilitation programmes or taking part in clinical trials. Furthermore, most of the study groups consisted of patients with severe airflow obstruction, which made it impossible to study the interaction between nutritional depletion, lung function and muscle function. To our knowledge, no data have been published on the prevalence of nutritional depletion in a random out-patient COPD population.

Nutritional depletion is generally based on body weight expressed as a percentage of ideal weight. Body weight, however, does not take into account differences in body composition among individuals. Body weight consists of fat-mass, body cell mass (BCM) and extracellular water. Body cell mass reflects the quantity of actively metabolizing (liver, gut, immune system) and contracting (muscle) tissue [9]. Approximately $60 \%$ of the BCM consists of muscle. It is difficult to measure BCM in clinical practice. However, it is generally acknowledged that in the absence of excessive fluid shifts a useful estimate of BCM is given by the fat-free mass (FFM). Under this assumption, bioelectrical impedance can be considered as a quick, easy and reliable technique for the assessment of FFM [10].

In an earlier study, we investigated body composition in a large group of patients with severe airflow obstruction using bioelectrical impedance measurement, and reported that depletion of FFM may also occur in normal weight COPD patients [8]. These patients suffered from physical impairment, assessed by respiratory muscle strength and exercise performance, even to a greater degree than underweight patients showing a relative 
preservation of FFM. The aim of the present study was to investigate tissue depletion in relation to physiological function measured by lung function, respiratory and peripheral skeletal muscle function, in a random outpatient population with COPD.

\section{Methods}

\section{Patients}

The study group consisted of adult out-patients, recruited over 4 months on attending the lung function laboratory of the University Hospital Maastricht for routine lung function measurement and followed up at the out-patient department for COPD. These patients were assessed at first presentation. None were part of a rehabilitation programme. All patients included in this study had to fulfil criteria of breathlessness and chronic airflow limitation, defined as a measured forced expiratory volume in one second $\left(\mathrm{FEV}_{1}\right)$ lower than the $95 \%$ confidence interval of the $\mathrm{FEV}_{1}$ reference [11], and no significant $(>10 \%$ of the reference value) improvement after inhalation of a $\beta_{2}$-agonist. Patients were excluded if their health was impaired primarily by some disease process other than their lung disease, such as malignant disease, recent surgery, severe endocrine disorders or sarcoidosis, or if they had a suspected abnormal fluid balance manifested by the presence of oedema or regular use of diuretics, to avoid interference with the precision of the nutritional measures. Patients with bronchiectasis or other chronic pulmonary diseases were also excluded from the study.

\section{Nutritional assessment}

Body height and weight were measured with the patient wearing indoor clothing and without shoes. Body height was measured with the patient barefoot and standing, and was determined to the nearest $0.5 \mathrm{~cm}$. Body weight was measured with a beam scale to the nearest $0.1 \mathrm{~kg}$ (Seca, FRG). Frame size (small, medium or large) was calculated from wrist circumference [12]. Body composition was assessed using the single frequency bioelectrical impedance method [10]. FFM was calculated using a patient specific equation based upon a linear relationship

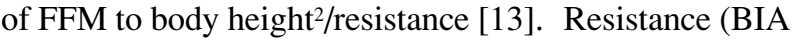
101/s RJL-Systems ${ }^{\circledR}$, Detroit, MI, USA) was measured with the patient in supine position, at both sides of the body [13]. The mean of the two resistance values was used for analysis. Body weight and FFM were expressed as a percentage of ideal body weight (PIBW) (FFMPIBW). Ideal body weight was determined as the mid-point of the weight range for a given height and frame size from the 1983 Metropolitan Life Insurance tables [14].

\section{Physiological function}

$\mathrm{FEV}_{1}$ and forced vital capacity (FVC) were measured with a wet spirometer (Gould Godart, Pulmonet III ${ }^{\circledR}$ ), with the highest value from at least three technically acceptable spirometric manoevres being used. Functional residual capacity, intrathoracic gas volume (ITGV) and airway resistance (Raw) were assessed in a body plethysmograph (Jaeger GMBH, Würzburg, Germany). Transfer factor for carbon monoxide (TLCO) was measured using a single-breath method. An estimate of alveolar volume (VA) was made by subtracting estimated dead space from inspiratory volume, and the TLCO was then corrected for alveolar volume (transfer coefficient KCO). The lung function variables were expressed as percentage of the reference values [11]. Maximal inspiratory (PImax) and expiratory (Pemax) mouth pressures were assessed as indicators of respiratory muscle strength using the technique described by Black and НyatT [15]. Pimax was measured when the patient maximally inspired at total lung capacity (TLC). PEmax was measured when the patient maximally exhaled to residual volume (RV). Handgrip-strength was assessed as a measure of peripheral skeletal muscle strength. A handgrip-dynamometer was used to determine the isometric grasp in each hand by measuring the maximally developed strength of the flexors of the fingers. The mean value of left and right hand strength was used for statistical analysis.

\section{Statistical analysis}

Descriptive statistics are given as mean (SD). FFMPIBW was related to PIBW and gender by multiple linear regression analysis. Since PIBW $<90 \%$ is a commonly employed clinical definition of nutritional depletion (table 1), this value and the corresponding gender adjusted value

Table 1. - Measures of physiological function categorized by body composition

\begin{tabular}{lrlrl}
\hline & \multicolumn{2}{c}{ Depletion* } & \multicolumn{2}{c}{ Normal** $^{*}$} \\
\hline $\mathrm{n}$ & \multicolumn{2}{c}{15} & \multicolumn{2}{c}{57} \\
Sex M/F & \multicolumn{2}{c}{$9 / 6$} & \multicolumn{2}{c}{$46 / 11$} \\
Age yrs & 64 & $(15)$ & 61 & $(13)$ \\
Weight kg & 54.8 & $(7.4)^{\dagger}$ & 77.9 & $(10.7)$ \\
FFM kg & 41.9 & $(5.8)^{\dagger}$ & 54.9 & $(6.5)$ \\
PIBW \% & 85.2 & $(7.4)^{\dagger}$ & 110.4 & $(12.5)$ \\
FFMPIBW \% & 65.1 & $(4.2)^{\dagger}$ & 77.6 & $(6.2)$ \\
PIMAX kPa & -6.9 & $(2.1)$ & -7.6 & $(2.2)$ \\
PEMAX kPa & 8.2 & $(2.2)$ & 8.7 & $(2.8)$ \\
Handgrip strength kg & 46.0 & $(27.2)^{\# \#}$ & 77.1 & $(29.8)$ \\
IVC \% pred & 82.1 & $(16.5)$ & 87.4 & $(17.9)$ \\
FEV \% pred & 45.5 & $(15.1)^{\#}$ & 55.2 & $(14.0)$ \\
KCO \% pred & 64.9 & $(16.2)^{\dagger}$ & 81.9 & $(24.5)$ \\
ITGV \% pred & 113.2 & $(22.5)$ & 110.2 & $(24.7)$ \\
RV \% pred & 120.7 & $(37.0)$ & 118.1 & $(32.6)$ \\
TLC \% pred & 95.3 & $(17.5)$ & 96.0 & $(14.2)$ \\
Raw \% pred & 239.2 & $(113.0)$ & 206.7 & $(103.6)$ \\
\hline
\end{tabular}

Data are presented as mean and SD in parenthesis. *: PIBW $<90 \%$ and/or FFMPIBW <67/69\%; **: FFMPIBW $\geq 67 / 69 \%$ and PIBW $\geq 90 \%$. FFM: fat-free mass; PIBW: body weight expressed as percentage of ideal body weight; FFMPIBW: fat-free mass expressed as percentage of ideal body weight; PImax: maximal inspiratory mouth pressure; PEmax: maximal expiratory mouth pressure; IVC: inspiratory vital capacity; Kco: transfer factor for carbon monoxide; ITGV: intrathoracic gas volume; RV: residual volume; TLC: total lung capacity; Raw: airway resistance. Difference between depleted and normal group: \#: $\mathrm{p}<0.04$; \#\#: $\mathrm{p}<0.01$; $: \mathrm{p}<0.001$. 
on the regression equation for FFMPIBW (69\% (males), $67 \%$ (females)) were taken for stratification. In this way, four groups could be formulated: 1) underweight patients with a depleted FFM; 2) underweight patients with a normal FFM; 3) normal weight patients with a depleted FFM; 4) normal weight patients with a normal FFM. Normal weight: body weight $\geq 90 \%$ of ideal weight; underweight: body weight $<90 \%$ of ideal weight; normal FFM: fat-free mass $\geq 69 \%$ (males) $/ 67 \%$ (females) of ideal weight; depleted FFM: fat-free mass $<69 \%$ (males)/67\% (females) of ideal weight.

Groups 1-3 were considered to suffer from tissue depletion. Comparison between groups was made using analysis of variance. A second comparison was done by the KCO, which may discriminate between emphysematous $(<60 \%$ of pred) and bronchitic ( $>80 \%$ of pred) patients [16].

\section{Results}

The study group consisted of 72 out-patients, suffering from a moderate degree of airflow obstruction (mean (sD) $\mathrm{FEV}_{1} 53$ (15) \% pred). Mean age of the 55 men and 17 women was 62 (13) yrs. Maintenance medication in this population consisted of theophyllines (43\%), $\beta_{2}$ sympathicomimetics $(41 \%)$, anticholinergics $(35 \%)$, and inhaled corticosteroids $(46 \%)$. Fourteen percent of the patients used oral corticosteroids $\left(<10 \mathrm{mg} \cdot\right.$ day $\left.^{-1}\right)$.

According to our definition of nutritional depletion, a substantial number of the study population (21\%) suffered from tissue depletion (table 1). Forty seven percent of the depleted and $13 \%$ of the nondepleted patients exhibited recent (within 6 months) involuntary weight loss. Figure 1 displays the distribution of the patients by body composition into the four groups. Fourteen percent of the patients were characterized by body weight loss and depletion of fat-free mass, whereas $7 \%$ had either weight loss or a depleted fat-free mass. When expressed as a percentage of the reference value, a significantly lower KCO $(\mathrm{p}=0.008)$ was found in the depleted group, but no difference in ITGV volumes and Raw (table 1). Although $\mathrm{FEV}_{1}$ was significantly lower $(\mathrm{p}<0.04)$ in the

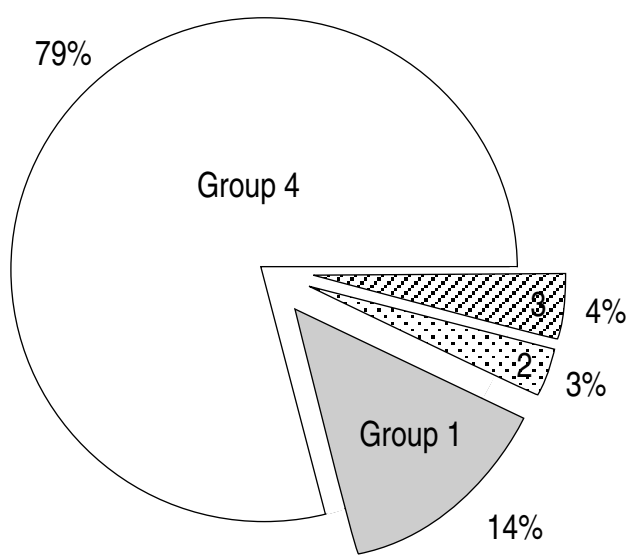

Fig. 1. - Characterization of the study group by body weight and fat-free mass (FFM). Group 1: $\square$ : underweight, depleted FFM; Group 2: $\because \because$ : underweight, normal FFM; Group 3: $\mathscr{Q}$ : normal weight, depleted FFM; Group 4: $\square$ : normal weight, normal FFM.

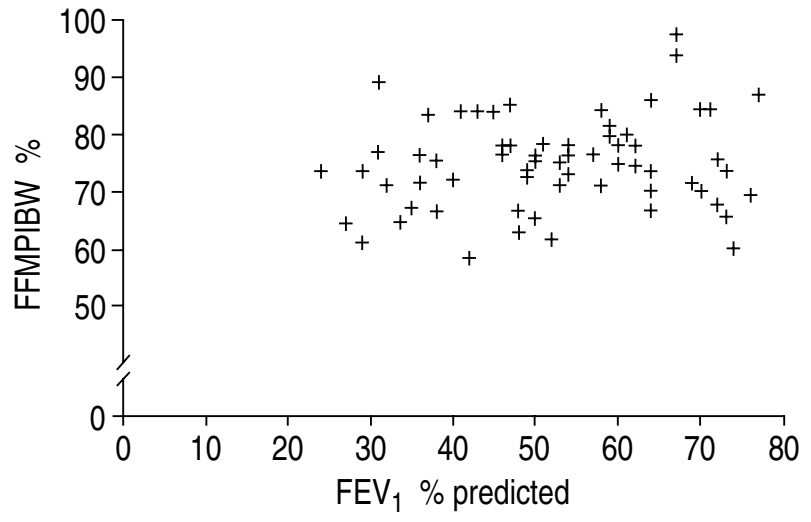

Fig. 2. - $\mathrm{FEV}_{1}$ (\% pred) plotted against FFMPIBW. No significant relationship can be found. FFMPIBW: fat-free mass expressed as a percentage of ideal body weight; $\mathrm{FEV}_{1}$ : forced expiratory volume in one second.

depleted group, the significance was lost when adjusted for the difference in Kco. The relationship between $\mathrm{FEV}_{1}$ and FFMPIBW is shown graphically in figure 2. Measures of muscle strength were lower in the depleted group, but only the difference in handgrip strength reached statistical significance $(\mathrm{p}<0.01)$.

Thirty eight percent of the patients with a $\mathrm{KCO}<60 \%$ pred (mean value 53.6 (6.8) \% pred) compared with 5\% of the patients with a $\mathrm{KCO} \geq 80 \%$ pred (mean value 99.1 (15.2) \% pred) were considered depleted $(p=0.009)$. Stratification by the transfer factor further revealed significantly higher values for ITGV, inspiratory vital capacity (IVC), TLC and RV in the group with a KCO $<60 \%$, whereas the degree of airway obstruction and the airway resistance were not significantly different between the groups (table 2). Mouth pressures were lower in the group with a low Kco, but statistical significance was not reached ( $\mathrm{p}=0.083$ for PImax).

Analysis of covariance revealed a significant effect of the KCO on peripheral skeletal muscle strength $(\mathrm{p}<0.01)$, even after adjustment for spirometry or ITGV. Analysis of co-variance, taking FFMPIBW as co-variate, indicated a significant independent contribution of KCO on PImax $(\mathrm{p}<0.05)$ but not on peripheral skeletal muscle strength.

Table 2. - Body composition and lung function categorized by the transfer factor

\begin{tabular}{lrlrl}
\hline & \multicolumn{2}{c}{ KCO $<60 \%$} & \multicolumn{2}{c}{ KCO $>80 \%$} \\
\hline Depletion* yes/no & \multicolumn{2}{c}{$6 / 10^{\# \#}$} & \multicolumn{2}{c}{$1 / 21$} \\
PIBW \% & 96.4 & $(12.9)^{\# \#}$ & 108.7 & $(11.9)$ \\
FFMPIBW \% & 70.1 & $(6.5)^{\dagger}$ & 78.8 & $(6.5)$ \\
IVC \% pred & 95.0 & $(14.7)^{\# \#}$ & 81.0 & $(16.4)$ \\
FEV \% pred & 50.2 & $(13.7)$ & 57.1 & $(14.4)$ \\
Raw \% pred & 237.2 & $(90.2)$ & 194.8 & $(119.9)$ \\
ITGV \% pred & 121.5 & $(22.7)^{\dagger}$ & 88.0 & $(22.5)$ \\
RV \% pred & 127.0 & $(33.2)^{\dagger}$ & 107.4 & $(36.7)$ \\
TLC \% pred & 103.9 & $(10.8)^{\dagger}$ & 87.4 & $(14.6)$ \\
PImax kPa & -6.8 & $(1.9)$ & -8.2 & $(2.5)$ \\
PEmax kPa & 8.4 & $(2.0)$ & 9.3 & $(2.3)$ \\
\hline
\end{tabular}

Data are presented as mean and SD in parenthesis. *: Chisquared test. Difference between emphysematous (Kco $<60 \%)$ and bronchitic $(\mathrm{KCO}>80 \%)$ patients. \#\#: $\mathrm{p}<0.01 ; \quad$ : $\mathrm{p}<0.001$. For abbreviations see legend to table 1 . 


\section{Discussion}

A compromised nutritional state is a common problem in patients with end-stage COPD. To our knowledge, no data are available regarding the prevalence and consequences of tissue depletion in a random out-patient population with less severe airflow obstruction. Over four consecutive months, we assessed body composition in all COPD patients admitted to the lung function laboratory of the University Hospital Maastricht and fulfilling the entry criteria. Tissue depletion was found in $20 \%$ of this group of stable out-patients with moderate airflow obstruction, whilst $20 \%$ had experienced recent involuntary weight loss. Compared to our previous study in severe COPD patients, depletion of FFM despite a normal body weight was found in a smaller group of patients. The minority of patients receiving oral corticosteroids did not differ in body composition and muscle function from the group not on steroid treatment.

Nutritional depletion is thus only a substantial problem when it is reflected in functional impairment. We therefore investigated the suggested relationship between tissue depletion and respiratory and peripheral skeletal muscle function. Depleted patients exhibited lower values for respiratory and peripheral skeletal muscle strength than nondepleted patients, but the difference was most pronounced for peripheral skeletal muscle strength. This is not a surprising finding if the decreased muscle strength is primarily determined by decreased muscle mass, since respiratory muscles comprise only a small proportion of total muscle mass. Another possibility could be a selective sparing of the respiratory muscles from muscle wasting. Recent studies in severe COPD patients suggest that, in addition to and independent of the loss of muscle mass, oxidative muscle metabolism may be impaired [17-19]. Several studies have tried to improve muscle function in end-stage COPD patients by nutritional supplementation, but have failed to show a clinically significant effect. The results of this study indicate that additional intervention studies should be conducted, particularly in this group of patients with moderate airflow obstruction, to investigate the relative effect of fat-free mass gain on the improvement of respiratory and peripheral skeletal muscle strength.

The depleted patients did not show a pronounced difference in spirometry or intrathoracic gas volumes from the nondepleted patients, indicating that weight loss and nutritional depletion is not confined to patients with severe airflow obstruction. The transfer coefficient (KCO), however, was found to be significantly lower in the group with tissue depletion. Stratification by Kco, which may discriminate between emphysematous and bronchitic patients $[16,20]$, also appeared to be a discriminant for the prevalence of tissue depletion. The relationship between emphysema (based on measurements of diffusion capacity) and nutritional depletion has been reported by some authors, but disputed by others $[4,5]$. This controversy may be explained by the patient groups studied and the criteria to define emphysema [20]. The significantly higher values for ITGV, IVC, TLC and RV in the group with lower KCO compared with the group with a
KCO $>80 \%$ supports emphysematous changes in the depleted group, whereas airflow obstruction assessed by spirometry and body plethysmographic measurement of airway resistance, was not different between the groups.

Contrary to other studies, our definition of tissue depletion in COPD was based not only on the relative size of body weight, but also included the size of the fat-free mass. In agreement with our earlier study in severely obstructive COPD patients [8], we found that FFMPIBW was a better predictor of respiratory and peripheral skeletal muscle strength than PIBW.

In conclusion, this study indicates that nutritional depletion is a significant problem not only in patients with severe COPD, but also in COPD out-patients with moderate airflow obstruction, especially in combination with an impaired diffusion capacity. The results further suggest that an abnormal body composition should be considered in the analysis of impaired respiratory and peripheral skeletal muscle strength in these patients. The lung function characteristics of the depleted group emphasize the role of the integrity of the alveolocapillary membrane in the development of tissue depletion. Further studies are needed to determine the contribution of this lung function impairment in the pathogenesis of somatic wasting in COPD patients.

\section{References}

1. Vandenbergh E, Van de Woestijne K, Gyselen A. Weight changes in the terminal stages of chronic obstructive lung disease. Am Rev Respir Dis 1967; 95: 556-566.

2. Wilson DO, Rogers RM, Wright E, Anthonisen NR. Body weight in chronic obstructive pulmonary disease. Am Rev Respir Dis 1989; 139: 1435-1438.

3. Driver AG, McAlevy MT, Smith JL. Nutritional aspects of patients with chronic obstructive pulmonary disease and acute respiratory failure. Chest 1982; 82: 568571.

4. Braun SR, Keim NL, Dixon RL, et al. The prevalence and determinants of nutritional changes in chronic obstructive pulmonary disease. Chest 1984; 86: 558-563.

5. Openbrier DR, Irwin MM, Rogers RM, et al. Nutritional status and lung function in patients with emphysema and chronic bronchitis. Chest 1983; 87: 17-22.

6. Fiaccadori E, Del Canale S, Coffrini E, et al. Hypercapnic-hypoxemic chronic obstructive pulmonary disease (COPD): influence of severity of COPD on nutritional status. Am J Clin Nutr 1988; 48: 680-685.

7. Gray-Donald K, Gibbons L, Shapiro SH, Martin JG. Effect of nutritional status on exercise performance in patients with chronic obstructive disease. Am Rev Respir Dis 1989; 140: 1544-1548.

8. Schols AMWJ, Soeters PB, Dingemans AMC, et al. Prevalence and characteristics of nutritional depletion in patients with stable COPD eligible for pulmonary rehabilitation. Am Rev Respir Dis 1993; 147: 11511156.

9. Forse RA, Shizgal HM. Serum albumin and nutritional status. J Parent Ent Nutr 1980; 4: 450-454.

10. Lukaski HC, Johnson PE, Bolonchuk WW, Lykken GI. Assessment of fat-free mass using bioelectrical impedance measurements of the human body. Am J Clin Nutr 1985; 41: 810-817. 
11. Quanjer PH ed. Standardized lung function testing. Bull Eur Physiopathol Respir 1983; 19: 7-44.

12. Shronts EP. Nutritional support dietetics; core curriculum 1989. American Society for Parenteral and Enteral Nutrition, Minneapolis, Minnesota, 1989.

13. Schols AMWJ, Wouters EFM, Soeters PB, Westerterp KP. Body composition by bioelectrical impedance analysis compared to deuterium dilution and skinfold anthropometry in patients with chronic obstructive pulmonary disease. Am J Clin Nutr 1991; 53: 421-424.

14. Metropolitan Life Insurance Company. New weight standards for men and women. Statist Bull Metrop Life Found 1983; 64: 1-4.

15. Black LF, Hyatt RE. Maximal respiratory pressures: normal values and relationship to age and sex. Am Rev Respir Dis 1969; 99: 696-702.

16. Gelb AF, Gold WM, Wright RR, Bruch HR, Nadel JA.
Physiologic diagnosis of subclinical emphysema. Am Rev Respir Dis 1983; 107: 50-63.

17. Del Canale $\mathrm{S}$, Soderlung K, Fiaccadori E, et al. Abnormal energy metabolism in severe chronic obstructive lung disease (COLD). Eur J Respir Dis 1986; 69 (Suppl. 146): 239-243.

18. Tada H, Kato H, Musawa $\mathrm{T}$, et al. ${ }^{31} \mathrm{P}$-nuclear magnetic resonance evidence of abnormal skeletal muscle metabolism in patients with chronic lung disease and congestive heart failure. Eur Respir J 1992; 5: 163-169.

19. Wuyam B, Payen JF, Levy P, et al. Metabolism and aerobic capacity of skeletal muscle in chronic respiratory failure related to chronic obstructive pulmonary disease. Eur Respir J 1992; 5: 157-162.

20. Gelb AF, Schein M, Kuei J, et al. Limited contribution of emphysema in advanced chronic obstructive pulmonary disease. Am Rev Respir Dis 1993; 147: 1157-1161. 